\title{
New Prediction Model for Cardiac Arrest Time of Palliative Care Patients in the Intensive Care Unit: A Single Center Retrospective Cohort Study
}

Shunsuke Takaki ( $\sim$ shunty5323@gmail.com )

Yokohama City University Hospital

Research article

Keywords: Death prediction, Autonomic nervous system, continuous monitoring, Palliative Care

Posted Date: September 15th, 2020

DOI: https://doi.org/10.21203/rs.3.rs-66184/v1

License: (c) (1) This work is licensed under a Creative Commons Attribution 4.0 International License.

Read Full License 


\section{Abstract}

Background: The trend of population ageing is a significant health issue in Japan and palliative care and end of life care are becoming key components of medical care. In the terminal care setting, the ability to accurately predict the time of death is an important research objective because it may allow family members to share valuable moments with the patient before death. Previous studies have investigated the time to death following withdrawal of therapy and several predictors of the time of death have been defined. The aim of this study was to determine an algorithm for the prediction of cardiac arrest time in the palliative care setting.

Method: We retrospectively collected data of patients in the Yokohama City University Hospital between 2010 and 2016. Vital signs were collected until cardiac arrest, with continuous blood pressure recordings of $<80 \mathrm{mmHg}$. We hypothesized that a breakdown of the autonomic nervous system was close to the time of cardiac arrest. We used the shock index (SI) defined as heart rate divided by systolic blood pressure (SBP) as the ratio variable for determination of the terminal phase.

Results: A total of 4330 patients were admitted to the ICU. Of these, 19 patients were included to this study. We developed an exponential decay model using the SI and SBP: predicted Shock Index $=0.995+$ (6.931-0.995) $\mathrm{e}^{-0.035 \times \mathrm{SBP}}$ AUC 0.650 (0.512 to 0.788). The disparity between the real heart rate and predicted hear rate was $-10 \mathrm{bpm}$ (sensitivity, $49.9 \%$; specificity, $75.8 \%$; likelihood ratio, 2.06 ). In the validation data set, sensitivity was $52.7 \%$, specificity was $79.8 \%$, PPV was $35.7 \%$, and NPV was $88.8 \%$. LR was 2.61. Predicted residual time to cardiac arrest was calculated as follows: $12.3+(0.18 \mathrm{x}$ age $)+$ (male: -6.0 , female: 0$)+($ heart rate $\times 0.65)+(0.28 \times$ Systolic Blood Pressure $)+(0.34 \times$ Heart Rate $)+(-0.25$ $x$ Respiratory Rate).

Conclusions: The new prediction model estimates the breakdown of the autonomic nervous system within 120 minutes of cardiac arrest. These algorithms might improve palliative care for last timing in the terminal situation.

\section{Background}

The trend of population ageing is a significant health issue in Japan and palliative care and end of life care are becoming key components of medical care. In the terminal care setting, the ability to accurately predict the time of death is an important research objective because it may allow family members to share valuable moments with the patient before death. Previous studies have investigated the time to death following withdrawal of therapy and several predictors of the time of death have been defined. 1,2 To date, the sensitivity and specificity of predictive variables are insufficient to predict the exact time of death and further research is required to develop a predictive model. ${ }^{1,3}$ In the terminal setting, unexpected sudden or prolonged death may affect the terminal care process between involving the family and the patient and cause difficulties with the process of organ donation after circulatory death. Therefore, it is important to develop an accurate model for predicting the time of death in the palliative care and terminal 
care setting. Many studies have developed prediction models using patient data sets, although no model with a high sensitivity have been reported to date. In our hypothesis, low sensitivity and specificity were caused by patient data sets. The majority of studies used one set of data derived from one patient for the prediction model at an arbitrary timing. One data set per patient may be insufficient for the prediction of death, because cardiac function, the autonomic nervous system, and backgrounds vary according to individual patients. Several studies used heart rate variability for the prediction of the time of death $4,5 \mathrm{In}$ this study, heart rate variability was measured every 30 minutes and researchers focused on the correlation between cardiac autonomic nerve system dysfunction and the prediction of the time of death. ${ }^{5}$ Other studies examined the variation of vital signs as a predictive factor for the final day of life of terminally ill patients. ${ }^{5}$ This study used patient vital signs for the prediction of the time of death and these were used twice per day. The change in vital signs had a low sensitivity (35\%) for the prediction of death, and the studies concluded that routine monitoring of vital signs was not recommended for the prediction of the time of death. These earlier studies did not use a continuous vital sign for predicting the time to death. We focused on parameters which were measured continuously in the palliative care situation. We hypothesized that heart rate and blood pressure discretely fluctuate because autonomic nerve system dysfunction occurs before cardiac arrest. Therefore, this discrete fluctuation in heart rate and blood pressure is considered the key point for the prediction of the time to death in the palliative care setting. The aim of this study was to develop a new prediction model for the time to death with included individual fluctuations in the continuous measurements of vital signs during deterioration in the palliative care situation. A new algorithm may allow patients and family members to share their last moments together before cardiac arrest and make effective use of intensive care beds.

\section{Methods}

\section{Study design}

This study was approved by the institution review board of Yokohama City University Hospital (Ref. B170200037), and was registered with the University Medical Information Network (UMIN ID: 000028958). We retrospectively collected data of patients admitted to the intensive care unit (ICU) at Yokohama City University Hospital between 2010 and 2016 and diagnosed with cardiac arrest on the ICU, without transfer to the ward in the palliative care situation, which was determined by the doctor and defined as unresponsiveness to treatment for the primary disease and hemodynamic instability. Arterial line insertion allowed continuous measurement of vital signs until cardiac arrest. Biological monitoring (Nihon Koden central monitoring system CNS- $621^{\mathrm{R}}$ ) allowed the extraction of biological information data every 1 minute. The biological data included systolic blood pressure (SBP), diastolic blood pressure (DBP), mean blood pressure (MBP), heart rate, respiratory rate, temperature, oxygen saturation. These parameters were collected from hospital admission to admission to ICU and until the heart rate was recorded as 0 beats per minute. We identified the vital signs at the point of deterioration in the clinical status for analysis in the palliative care situation which was determined as unresponsiveness to treatment for primary disease and hemodynamic instability. A SBP of $<80 \mathrm{mmHg}$ for a duration of 10 
minutes was defined as a deterioration in the general condition of the patient and marked the onset of the end of life process. Vital signs recorded by the biological monitoring system normally contain artifact due to a number of factors, such as blood test sampling from arterial lines, nursing care, and restless activity at the cannulation site. Therefore, we manually checked the vital signs to establish whether these accurately represented the patient's condition. We excluded data considered inaccurate due to artifact. Each vital sign data was assigned a residual time which decreased progressively until the point of death defined by a heart rate of $0 \mathrm{bpm}$. Baseline demographics, including age, height, body weight, diagnosis, and duration of ICU admission, were also collected.

Our prediction method involves a two-step approach for the prediction of the time to death. The first step involves predicting the point in time at 120 minutes before cardiac arrest using a new algorithm and applied to biological data. Chiang, J. et. al reported association between cardiovascular autonomic functions and residual time to death with using heart rate variability ${ }^{4}$ Karimi, M. et.al. reported that cardiac nerve system dysfunction correlated with residual time to death. ${ }^{5}$ Therefore, we focused on cardiac nerve system dysfunction which results in an imbalance between heart rate and blood pressure and used this to develop an algorithm for the predicting the timing of 120 minutes before cardiac arrest. We determined a non-linear regression model for the prediction of death based on heart rate and blood pressure. The dispersion from the non-linear regression model was defined as the deterioration to cardiac arrest. The second step involved the calculation of the accurate time of death using the linear regression model. Therefore, we used a new prediction model to estimate cardiac arrest (primary outcome) and develop an algorithm for the prediction of residual time to death in the palliative care situation (secondary outcome).

\section{Statistical methods}

Descriptive patient data are expressed as median values and interquartile range. Initially, vital signs were divided into those occurring for a period over 120 minutes from cardiac arrest, described as volatile set (VS), and those occurring within 120 minutes of cardiac arrest, described as terminal set (TS). According to a scaling law for the training set to validation set ratio ${ }^{6}$, we randomly allocated $80 \%$ of patient vital sign data to the development set and $20 \%$ of data to the validation set in both the VS and TS. In the development set, we developed a new prediction model which predicted the death within 120 minutes of cardiac arrest using vital data extracted from biological monitoring. The new prediction model performed non-linear regression analysis with an exponential decay model. In the non-linear regression analysis, we used SBP and the shock index, defined as the heart rate divided by SBP, as the ratio variable. We compared the predicted heart rate calculated from the non-linear regression model and the real heart rate. In the development set, the threshold for disparity between the predicted heart rate and the real heart rate was determined to predict the time at 120 minutes before cardiac arrest.

The determination of the threshold was performed using the nonparametric $t$ test, the log rank test, and Receiver Operating characteristic (ROC) curve for the area under the curve. Linear regression analysis was 
used for the calculation of the accurate residual time to death in the terminal situation. We used Akaike's information criterion (AIC) stepwise procedure and selected the model with the lowest AIC value ${ }^{7}$. Age, sex, systolic blood pressure, respiratory rate, and heart rate were selected as confounding factors.

In the validation set, we analyzed the diagnostic power of the new prediction model and the algorithm for predicting residual time to death. We examined the difference between residual time to death using the linear regression model and the real residual time. The diagnostic power of the prediction model was calculated using the chi-square with Yates' correction.

For 2-tailed tests, $\mathrm{P}<0.05$ was considered statistically significant. Statistical analyses were performed with Prism 6 for Mac OS X 6.9b (GraphPad Software, San Diego, California) and R 2.13.0 statistical software (R Foundation for Statistical Computing, Vienna, Austria).

\section{Results}

\section{Participants}

A total of 4330 patients were admitted to the ICU between 2010 and 2016 and, of these, 32 patients were diagnosed with cardiac arrest on the ICU. Thirteen out of the 32 patients were excluded due to insufficient data and age $<18$ years old. Finally, 19 patients were included in the present study and 12,050 data sets representing vital signs were recorded. 12,038 data sets were enrolled in the present study and 12 data set were manually excluded due to artifact or measurement error made by clinician judgment. In the all data set, 9,915 out of 12,038 (82.3\%) data set of vital signs were allocated to VS and 2,111 of 12,038 $(17.7 \%)$ data set were allocated to TS. $80 \%$ of each data set, randomly selected 7932 data set from VS and 1,689 data set from TS were allocated to the development data set, respectively. Furthermore, in the development set, 6,243 randomly selected VS data sets out of a total of 7932 were used for non-linear regression analysis and 1,689 from each development data set (VS and TS) were used for determination of threshold. Also, $20 \%$ of each data set, 1,983 data set from VS and 422 data set from TS were allocated to the validation set. (Figure. 1) Subject demographic characteristics were expressed as median and interquartile range, as follows: age 63 years (53 to 75 years), sex (men 15/19, 79\%), duration of ICU stay 3 days ( 1 to 8 days), duration of hospital stay 7 days ( 3 to 23 days). The diagnoses were categorized as follows: 5 cardiac surgical(26\%), 4 surgical (21\%), 5 medical (26\%), and 5 cancer (26\%).

\section{Outcome data}

Median and interquartile range of residual time to death from the point when blood pressure was $<80$ $\mathrm{mmHg}$ was 279 minutes (126 to 864 ) across all data. Non-linear regression with an exponential decay model showed a fitted curve for the association between shock index and SBP. The exponential decay model is widely used in the natural sciences. ${ }^{8}$ In the development set, from the 6,243 variables, exponential decay was derived from the following equation. 


$$
y=\text { Plateau }+(\text { Y } 0 \text {-Plateau }) e^{-k x}
$$

The parameters of the equation were as follows: $Y 0=6.931$ (6.638 to 7.224), plateau $=0.995$ ( 0.926 to $1.063), \mathrm{K}=0.035$ (0.327 to 0.037$)$. The following equation was derived:

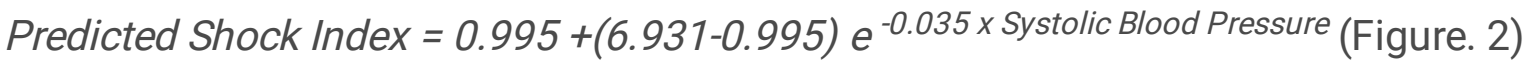

$\mathrm{R}$ square is 0.699 .

\section{Main results}

Median and interquartile range of the disparity in the two data sets are 1.90 (1.1 to 2.9) for the volatile data set and -9.8 (-10.9 to -8.5) for the terminal data set, respectively. Area under the ROC of the new prediction model in the development set was 0.650 (0.512 to 0.788$), p<0.001$, and the threshold of disparity between real and predicted heart rate was $-10 \mathrm{bpm}$ with sensitivity of $49.9 \%$, specificity of $75.8 \%$, and likelihood ratio of 2.06. (Figure. 3)

In the validation set, the predictive power of the new prediction model for cardiac arrest conducted in the development set was analyzed. In the validation data set, the diagnostic power was as follows: sensitivity $52.7 \%$ (47.8 to 57.6 ), specificity $79.8 \%$ (78.0 to 81.6 ), positive predictive value $35.7 \%$ (31.9 to 39.6 ), negative predictive value $88.8 \%$ (87.2 to 90.3$)$, and likelihood ratio 2.61 .

\section{Other analyses}

In the secondary outcome analysis, we determined a new formula for the accurate prediction of the residual time to cardiac arrest. Residual time to cardiac arrest was prescribed as the dependent variable. Age, sex, heart rate, respiratory rate, and SBP were prescribed as explanatory variables, measured within 120 minutes from cardiac arrest. Diastolic blood pressure and mean blood pressure were excluded, due to the strong correlation with SBP. The following formula was determined using the linear regression model:

Predicted Residual Time to Cardiac Arrest $=12.3+(0.18 \times$ Age $)+($ Male: -6.0 , Female: 0$)+(0.28 \times$ Systolic Blood Pressure $)+(0.34 \times$ Heart Rate $)+(-0.25 \times$ Respiratory Rate) (Table. 1$)$

\section{Table 1}

Residual time to cardiac arrest with linear regression model analyzed with vital data within 120 minutes from cardiac arrest in the development set.

Residual standard error: 28.87 on 1287 degrees of freedom Multiple R-squared: 0.3167, Adjusted Rsquared: 0.314 ; F-statistic: 119.3 on 5 and 1287 degrees of freedom, p-value: $<2.2 \mathrm{e}-16$ 


\begin{tabular}{|l|c|c|c|c|}
\hline Factor & Estimate & Std. Error & t-value & $\mathrm{p}$-value \\
\hline (Intercept) & $12.34(2.25-22.43)$ & 5.14 & 2.4 & 0.017 \\
\hline Heart Rate & $0.34(0.28-0.4)$ & 0.03 & 11.57 & $<0.001$ \\
\hline Respiratory Rate & $-0.25(-0.36--0.14)$ & 0.06 & -4.37 & $<0.001$ \\
\hline Systolic Blood Pressure & $0.28(0.18-0.37)$ & 0.05 & 5.81 & $<0.001$ \\
\hline Sex. Male.1, Female.0. & $-0.60(-4.56-3.37)$ & 2.02 & -0.3 & 0.77 \\
\hline Age & $0.18(0.06-0.29)$ & 0.06 & 2.9 & 0.004 \\
\hline
\end{tabular}

In the validation set, the disparity between real residual time to cardiac arrest and predicted residual time to cardiac arrest calculated with the linear regression model was expressed as mean (3.6 minutes), median (-1.49 minutes), and interquartile range (-22.2 to 31.4 minutes).

\section{Discussion}

Our new prediction model can estimate the residual time to death in the palliative care situation within 120 minutes from cardiac arrest with good diagnostic power (sensitivity $52.7 \%$, specificity $79.8 \%$, positive predictive value $35.7 \%$, and negative predictive value $88.8 \%$ ). The accuracy of our predictive value was good compared to the following studies: Lewis et al. reported sensitivity of $42 \%$ and specificity of $76 \%{ }^{9}$; Coleman et al. reported $39 \%$ sensitivity and $96 \%$ specificity ${ }^{10}$; and the DeVita et al. reporting sensitivity of $79 \%$ and specificity of $63 \%{ }^{11}$ A significant advantage of this study, which distinguishes it from previous research, is that we used a new prediction model based on continuous measurement of vital signs which were recorded every 1 minute. Brieva et al. reported that the evaluation of the ICU specialist is the clinical standard for predicting the time of death following withdrawal of cardiorespiratory support. ${ }^{12}$ The ICU specialist evaluation was implemented at sn arbitrary timing. However, our new prediction model, based on recordings performed every 1 minute, repeated the prediction for the timing of cardiac arrest. Therefore, our new prediction model outweighs the previous studies because of repeatedly prediction.

Previous reports, different parameters for the prediction of cardiac arrest have been reported; these include physiological data but also blood tests, past history, and background. 2,13,14 Our new prediction model was calculated using SBP and heart rate, which was automatically calculated using information communication technology. It is envisaged that our model will be widely used over the next in the different areas. Because these parameters are quite standard variables which widely measured at the chronic and acute care situation.

Another strength of this study was our hypothesis which focused on the breakdown of the autonomic nerve system. 
Mohammad et al. reported that the analysis of heart rate variability was a useful predictor for cardiac death. ${ }^{5}$ Heart rate variability was determined by the interaction between the sympathetic and parasympathetic autonomic nervous system. In our algorithm, blood pressure and heart rate were used as predictive parameters which represented the interaction between the sympathetic and parasympathetic autonomic nervous system. Heart rate variability should be interpreted with caution. ${ }^{15}$ breathing control is crucial to in order to accurately interpret HRV. ${ }^{15}$ Billman, G reported that heart rate variability was associated with mechanical events due to thoracic pressure changes and cardiac filling pressure during respiration. Furthermore, Chiang, J. et. al. reported cardiac arrhythmia and major cardiac surgery affect heart rate variability ${ }^{4}$ Therefore, in this study, we focused on the balance between blood pressure and heart rate, not on the heart rate variability. In the controlled cardiac nervous system, heart rate and blood pressure are interlocked due to sympathetic nerve system and parasympathetic nerve system. ${ }^{16}$ In the future studies, we will develop our new prediction model by targeting the individual and determining an equation based on the autonomic nerve system pattern of the individual patient. In our strong point, consecutive measurement of vital sign could repeatedly predict imbalance sympathetic nervous system and parasympathetic nervous system, therefore, we predicting model has good diagnostic power.

In the palliative care situation, it is important to allow family members sufficient time with the patient, enabling contact with the patient before postmortem changes occur. Predicting the time of death within 120 minutes of cardiac arrest in the urban setting allows most family members to reach the hospital before the patient's death. Furthermore, this information would help healthcare providers to plan patient care and manage family members.

As our secondary outcome, we determined an equation to predict the residual time to cardiac arrest. The disparity between real residual time to cardiac arrest and predicted residual time to cardiac arrest calculated with the linear regression model was expressed as mean (3.6 minutes), median (-1.49 minutes), and interquartile range (-22.2 to 31.4 minutes). It is a reasonable difference for the prediction of the residual time to cardiac arrest. The originality of our research lies in the fact that there are no previous reports accurately predicting the residual time to cardiac arrest. The accurate determination of the residual time to cardiac arrest would be of value to healthcare providers, allowing them to help family members to say their last goodbyes. However, it is important to discuss with the family members whether they wish to know the residual time to cardiac arrest or not. Attitudes towards death vary among individuals and may be affected by religious beliefs. Therefore, it is important to acknowledge that in the clinical situation a mathematical equation may interfere with dying process.

\section{Limitations}

There are several limitations to the present study. First, the small sample size of 19 patients, even large vital data sets which recorded every minute from one patient were collected. 
In our analysis, repeated measurements from different patients were included in one cluster for statistical analysis. Therefore, many data set collected from the same patient were analyzed as one individual data set. This is one of the limitations, and should be improved for increasing sample size of patients in the future study. In our center, patients diagnosed as terminal, with terminal condition defined as unresponsiveness to treatment for primary disease and hemodynamic instability, were transferred to the ward to allow them to have time with family members. Therefore, only a small number of patients remain on the ICU and suffer from cardiac arrest. Secondly, we defined 120 minutes as the threshold for allocation to the two groups, the volatile set and the terminal set. In the urban setting, family members could reach the hospital within 120 minutes. However, in the rural setting, 120 minutes may be insufficient to reach to the hospital. Therefore, it may be more appropriate to use different timeframe to differentiate the two groups. Thirdly, our prediction model required consecutive measurements of heart rate and blood pressure with invasive blood pressure monitoring. Outside the ICU setting, noninvasive blood pressure monitoring is commonly used to measure blood pressure. Therefore, future studies targeting non-ICU patients should select alternative variables, measurement interval, and monitoring device.

\section{Conclusions}

Our new predicting model for the determination of the residual time to cardiac arrest had good diagnostic power. In the palliative care setting, the new prediction model predicts the breakdown of the autonomic nerve system with fluctuation of heart rate and systolic blood pressure before cardiac arrest. Further research will be required to examine individual fluctuation patterns of hemodynamic parameters in the palliative situation before death.

Furthermore, it is important to develop a technique for noninvasive monitoring allowing the prediction of the residual time to cardiac arrest in different clinical situations.

Our findings were derived from the analysis of continuous measurements of biological data recorded in the ICU. Therefore, our findings may be of limited generalizability in other settings. New monitoring devices may be developed to allow continuous recording of vital signs which would allow the application of our algorithm to a range of clinical situations.

\section{Abbreviations}

SI: shock index, SBP: systolic blood pressure, DBP: diastolic blood pressure, MBP: mean blood pressure, VS: volatile set, TS: terminal

\section{Declarations}

\section{- Ethics approval and consent to participate}


This study was approved by the institution review board of Yokohama City University Hospital (Ref. B170200037), and was registered with the University Medical Information Network (UMIN ID: 000028958).

\section{- Consent for publication}

This study does not contain individual information, therefore, informed consent was waived.

\section{- Availability of data and material}

The datasets used and/or analysed during the current study are available from the corresponding author on reasonable request.

\section{- Competing interests}

None

\section{- Funding}

This research is funded by Ministry of Internal Affairs and Communications Strategic Information

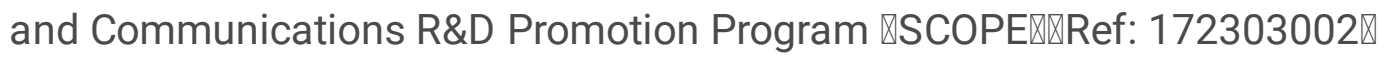

\section{- Authors' contributions}

T.S contributed to the study design, data collection, statistical analysis, and preparation of the manuscript.

\section{- Acknowledgements}

We thank all the research staff and the healthcare provider who supported our research.

\section{References}

1. Munshi $L$, et al. Predicting time to death after withdrawal of life-sustaining therapy. Intensive Care Med. 2015;41:1014-28.

2. Shapiro NI, et al. Mortality in Emergency Department Sepsis (MEDS) score: A prospectively derived and validated clinical prediction rule*. Crit Care Med. 2003;31:670-5. 
3. Cooke CR, Hotchkin DL, Engelberg RA, Rubinson L, Curtis JR. Predictors of time to death after terminal withdrawal of mechanical ventilation in the ICU. Chest. 2010;138:289-97.

4. Chiang J-K, Koo M, Kuo TBJ, Fu C-H. Association Between Cardiovascular Autonomic Functions and Time to Death in Patients With Terminal Hepatocellular Carcinoma. J Pain Symptom Manage. 2010;39:673-9.

5. Karimi Moridani M, Setarehdan SK, Motie Nasrabadi A, Hajinasrollah E. Non-linear feature extraction from HRV signal for mortality prediction of ICU cardiovascular patient. J Med Eng Technol. 2016;40:87-98.

6. Guyon I. A scaling law for the validation-set training-set size ratio. AT\&T Bell Lab 1-11 (1997). doi:10.1.1.33.1337.

7. Hiroji A 'Information theory and an extension of the maximum likelihood principle',. in 2nd International Symposium on Informtion Theory. Akademiai Kiado, Budapest. 267-281 (1973).

8. Leike A. Demonstration of the exponential decay law using beer froth. Eur J Phys. 2002;23:21-6.

9. Lewis J, et al. Development of the University of Wisconsin donation After Cardiac Death Evaluation Tool. Prog Transplant. 2003;13:265-73.

10. Coleman NL, Brieva JL, Crowfoot E. Prediction of death after withdrawal of life-sustaining treatments. Crit Care Resusc. 2008;10:278-84.

11. DeVita MA, et al. Donors after cardiac death: validation of identification criteria (DVIC) study for predictors of rapid death. Am J Transplant. 2008;8:432-41.

12. Brieva J, et al. Prediction of death in less than 60 minutes following withdrawal of cardiorespiratory support in ICUs. Crit Care Med. 2013;41:2677-87.

13. Morita T, Tsunoda J, Inoue S, Chihara S. Improved accuracy of physicians' survival prediction for terminally ill cancer patients using the Palliative Prognostic Index. Palliat Med. 2001;15:419-24.

14. Rady MY, Verheijde JL. Prediction of time to death after terminal withdrawal of life-support in nonheartbeating organ donation: Unaccounted variables and window of opportunity. Crit Care Med. 2012;40:986-8.

15. Billman GE. Heart rate variability - a historical perspective. Front Physiol. 2011;2:86.

16. Matsukawa T, Sugiyama Y, Mano T. Age-related changes in baroreflex control of heart rate and sympathetic nerve activity in healthy humans. J Auton Nerv Syst. 1996;60:209-12.

\section{Figures}




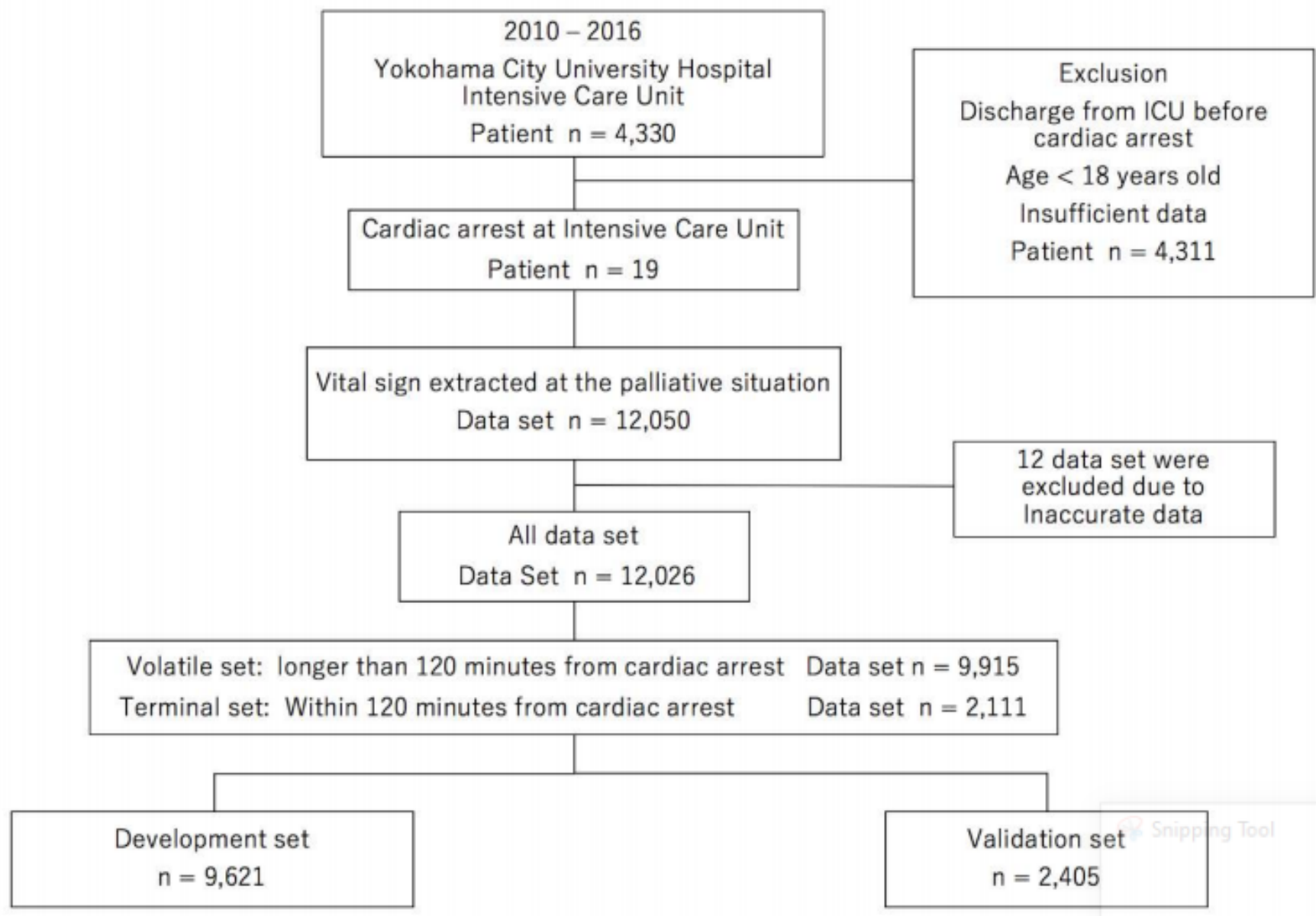

Figure 1

Process of enrollment and allocation to the development set or validation set. $80 \%$ of all I data sets were allocated to the development set $(9,621$ of 12,026$)$. The residual $20 \%$ of data sets were allocated to the validation set. $(2,405$ of 12,026$)$ 


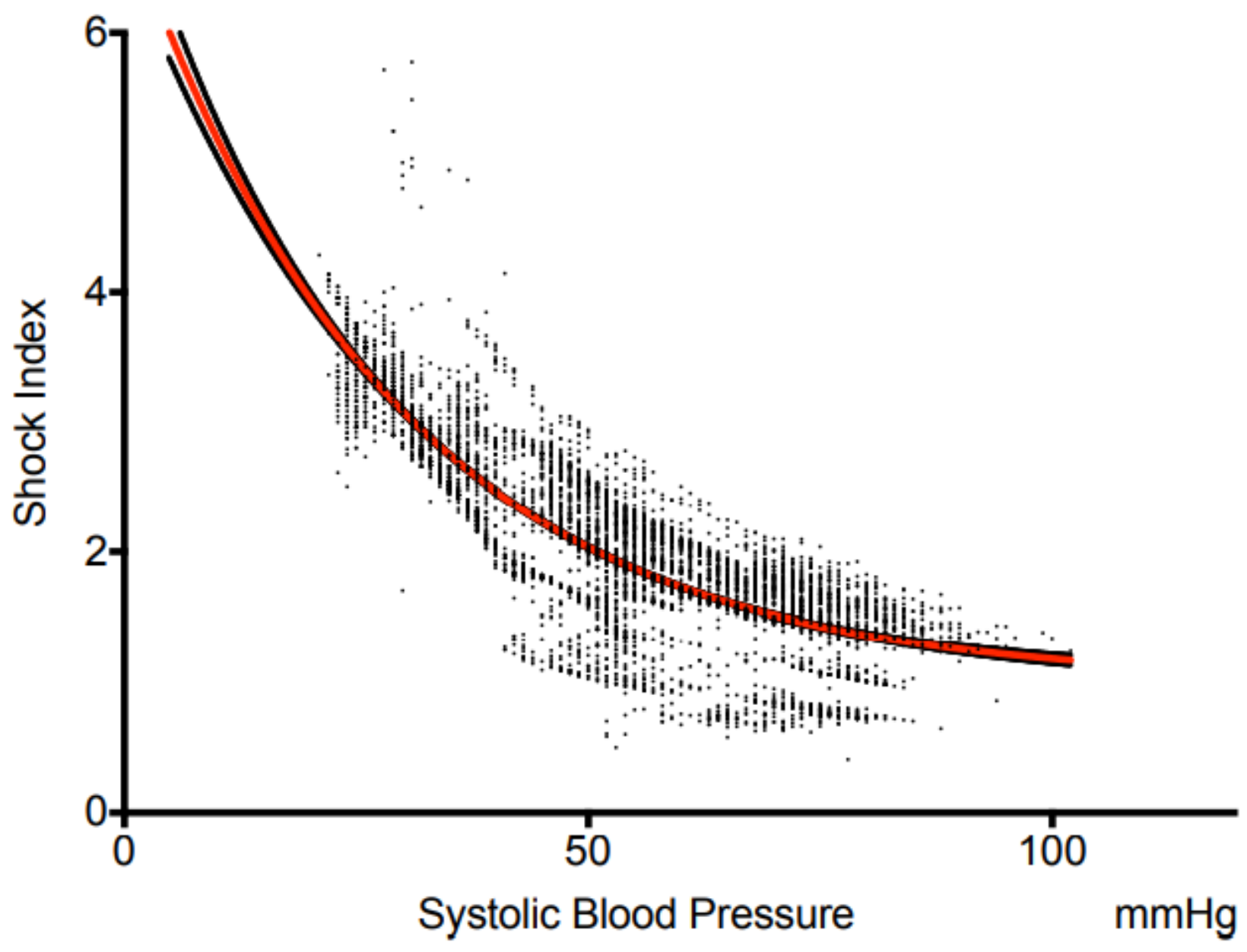

Figure 2

Plot of the association between shock index and systolic blood pressure in the volatile set showing vital signs from continuous low blood pressure to over 120 minutes from cardiac arrest. The red line of the non-linear regression curve is derived from the equation calculated in the development set:Predicted Shock Index $=0.995+(6.931-0.995)$ e $-0.035 \times$ Systolic Blood Pressure . R square is 0.699. 

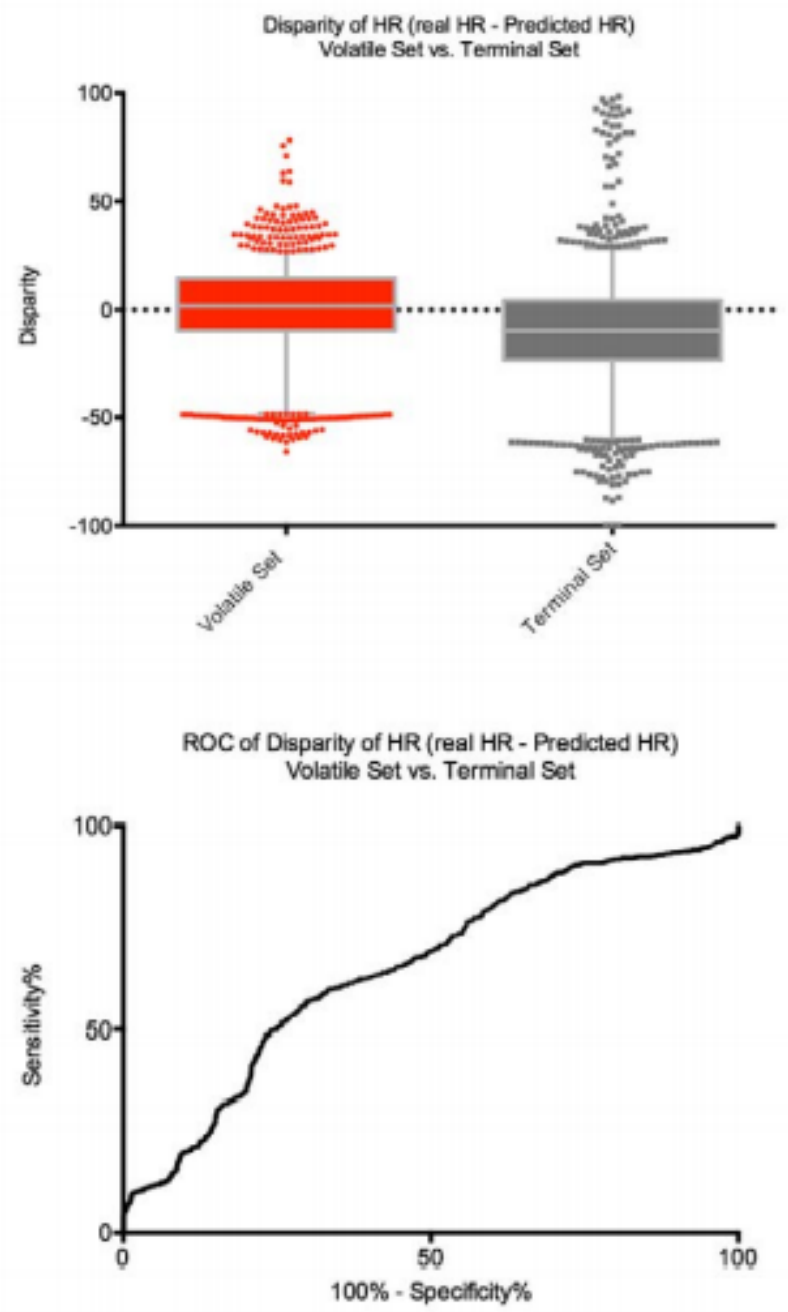

Figure 3

ROC curve of disparity between heart rates (real heart rate - predicted heart rate) between volatile set vs. terminal set. Volatile set: 1.90 (1.1 to 2.9) Terminal set: -9.8 (-10.9 to -8.5$) \mathrm{p}<0.001$ Area under the ROC curve Area: 0.650 , Standard Error: 0.070 95\% confidence interval: 0.512 to 0.788 , p value: $<0.001$ 